https://helda.helsinki.fi

\title{
Angiosarcome de la tête et du cou du sujet âgé
}

\section{Kluger, Nicolas}

2018

Kluger , N \& Lappalainen , K 2018 , ' Angiosarcome de la tête et du cou du sujet âgé ' , NPG

Neurologie - Psychiatrie - Gériatrie , vol. 18 , pp. 336-339 . https://doi.org/10.1016/j.npg.2018.06.005

http://hdl.handle.net/10138/307724

https://doi.org/10.1016/j.npg.2018.06.005

publishedVersion

Downloaded from Helda, University of Helsinki institutional repository.

This is an electronic reprint of the original article.

This reprint may differ from the original in pagination and typographic detail.

Please cite the original version. 


\title{
CAS CLINIQUE
}

\section{Angiosarcome de la tête et du cou du sujet âgé}

\section{Angiosarcoma on the head and neck in the elderly}

\author{
N. Kluger*, K. Lappalainen
}

Dermatology, Allergology and Venereology, University of Helsinki, Helsinki University Central Hospital, Meilahdentie 2, PO Box 16000029 HUS, Helsinki, Finlande

Disponible sur Internet le 29 juillet 2018

\section{MOTS CLÉS}

Angiosarcome ;

Scalp ;

Sujet âgé ;

Tumeur vasculaire
Résumé Les angiosarcomes sont des sarcomes des tissus mous rares d'origine endothéliale, mais agressifs et au pronostic sombre. L'angiosarcome de la tête et du cou de Wilson-Jones est une forme topographique particulière affectant principalement le scalp des sujets âgés. Nous rapportons un cas chez une patiente de 95 ans.

(c) 2018 Elsevier Masson SAS. Tous droits réservés.

\section{KEYWORDS}

Angiosarcoma;

Elderly;

Scalp;

Vascular tumor
Summary Angiosarcoma is a sub-type of soft-tissue sarcomas of endothelial origin. It is usually aggressive with poor prognosis. Angiosarcoma of the head and neck (Wilson-Jones) mainly affects the scalp among the elderly. We report the case of a 95-year-old woman.

(c) 2018 Elsevier Masson SAS. All rights reserved.

\footnotetext{
* Auteur correspondant.

Adresses e-mail : nicolas.kluger@hus.fi (N. Kluger), katriina.k.lappalainen@hus.fi (K. Lappalainen).
} 
Les angiosarcomes (AS) représentent $2 \%$ de tous les sarcomes des tissus mous et environ $5 \%$ des sarcomes cutanés sont des AS [1,2]. L'incidence des AS est en augmentation depuis 30 ans [1]. Ils touchent tous les âges, les deux sexes, et peuvent survenir dans n'importe quel tissu mou [1]. L'AS de la tête et du cou (AStc), aussi dénommé tumeur ou AS de Wilson-Jones [3] est la forme la plus fréquente d'AS, touchant avec prédilection l'homme âgé. Son diagnostic clinique peut être difficile. Nous rapportons ici un cas chez une patiente de 95 ans.

\section{Cas clinique}

Une femme de 95 ans était adressée en octobre 2016 en consultation pour l'évaluation d'une plaque violine du cuir chevelu d'aggravation progressive. Les antécédents comprenaient une maladie d'Alzheimer, une hypertension artérielle, une arythmie et une rectocolite ulcéro-hémorragique. La patiente n'avait aucun antécédent dermatologique particulier. Elle était en maison de retraite depuis le début de l'année. Au printemps 2016 avait été notée une discrète rougeur asymptomatique du scalp. Cette dernière s'était progressivement aggravée durant l'été avec une extension vers l'occiput ainsi que vers l'os temporal. Il n'existait pas de prurit, mais la patiente se plaignait d'une hypersensibilité du cuir chevelu lors des shampoings ou de la coiffure. À la demande d'une soignante, elle nous était finalement adressée pour évaluation. À l'examen, elle présentait une large nappe maculeuse, rouge foncée, " en casque ", aux bords bien délimités mais déchiquetés (Fig. 1). La nappe était mieux visible en périphérie à la lisière du cuir chevelu. On notait sur le vertex des lésions punctiformes plus sombres évocatrices de structures vasculaires. Enfin, sur l'occiput, on palpait une infiltration sous-cutanée mobile de $3 \times 2 \mathrm{~cm}$ (Fig. 2 ). Devant cette tache cutanée rouge sombre lie de vin étendue et d'extension progressive d'allure vasculaire avec un nodule du scalp chez une femme âgée, le diagnostic d'AS était évoqué en priorité. Deux biopsies au punch de $4 \mathrm{~mm}$ étaient réalisées sur la nappe et sur le nodule occipital. L'analyse histologique retrouvait des structures vasculaires nombreuses, irrégulières, disséquant le derme et avec une invasion périneurale (Fig. 3). La tumeur présentait un marquage vasculaire fortement positif (FLI-1 et CD31+, mais CD34-) (Fig. 4) et également lymphatique (D2-40+) ainsi qu'une forte activité mitotique (MIB-1, 70-80\% de cellules positives). L'infiltrat n'exprimait en revanche pas de marqueurs en faveur d'une maladie de Kaposi (HHV-8), d'un mélanome (S-100, Mart-1) ou d'un carcinome (CK-Pan). L'histologie et l'immunohistochimie confirmaient le diagnostic d'AS peu différencié. L'état général de la patiente s'aggravait rapidement dans le mois suivant et cette dernière décédait avant tout bilan d'extension et mise en route d'un traitement.

\section{Discussion}

Les facteurs de risque connus ne concernent qu'une partie des AS [1,2] : l'AS sur lymphœdème chronique congénital ou acquis (syndrome de Stewart-Treves), les

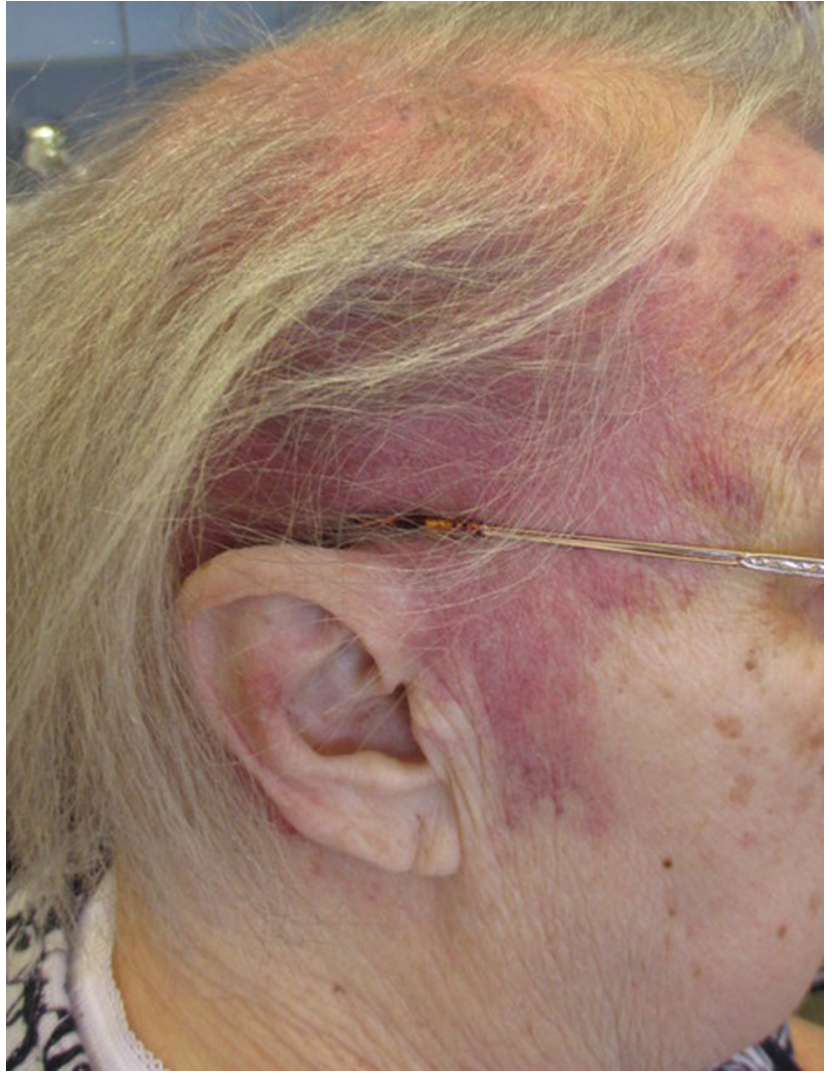

Figure 1. Nappe violine pariétale et temporale, aux contours bien délimités mais déchiquetés.

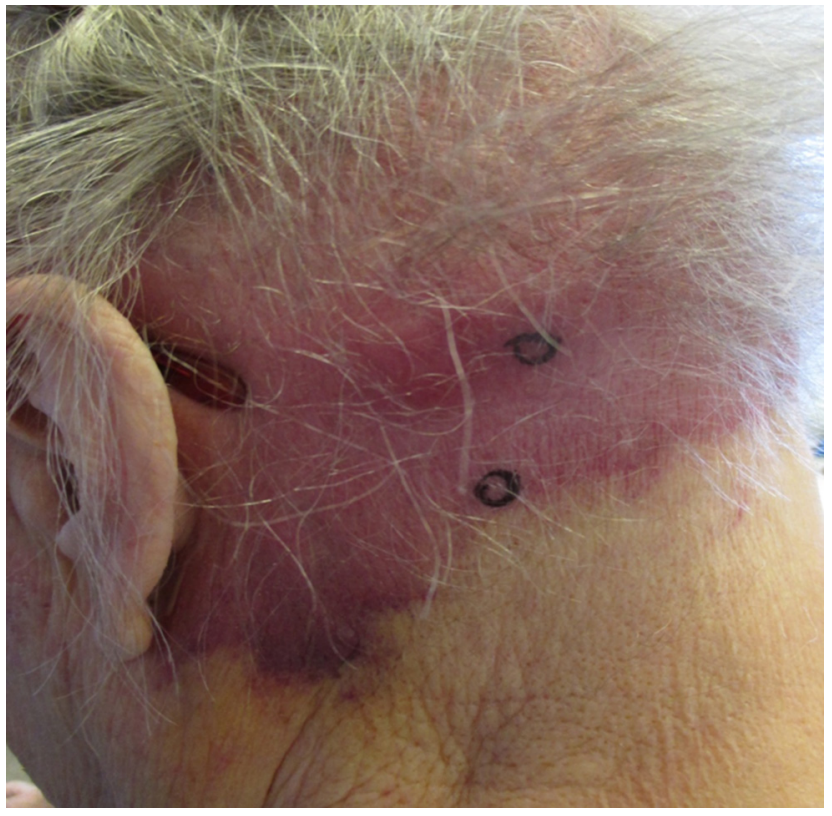

Figure 2. Nappe violine rétro-auriculaire et occipitale avec un nodule infiltré de $3 \mathrm{~cm}$. Les marques correspondent aux zones biopsiées.

AS post-radiothérapie (AS mammaire), les AS associés à certaines substances toxiques (arsenic, chlorure de vinyle, thorium...) et enfin certaines maladies (syndrome de Mafucci, syndrome de Klippel-Trenaunay-Weber) [1,2]. 


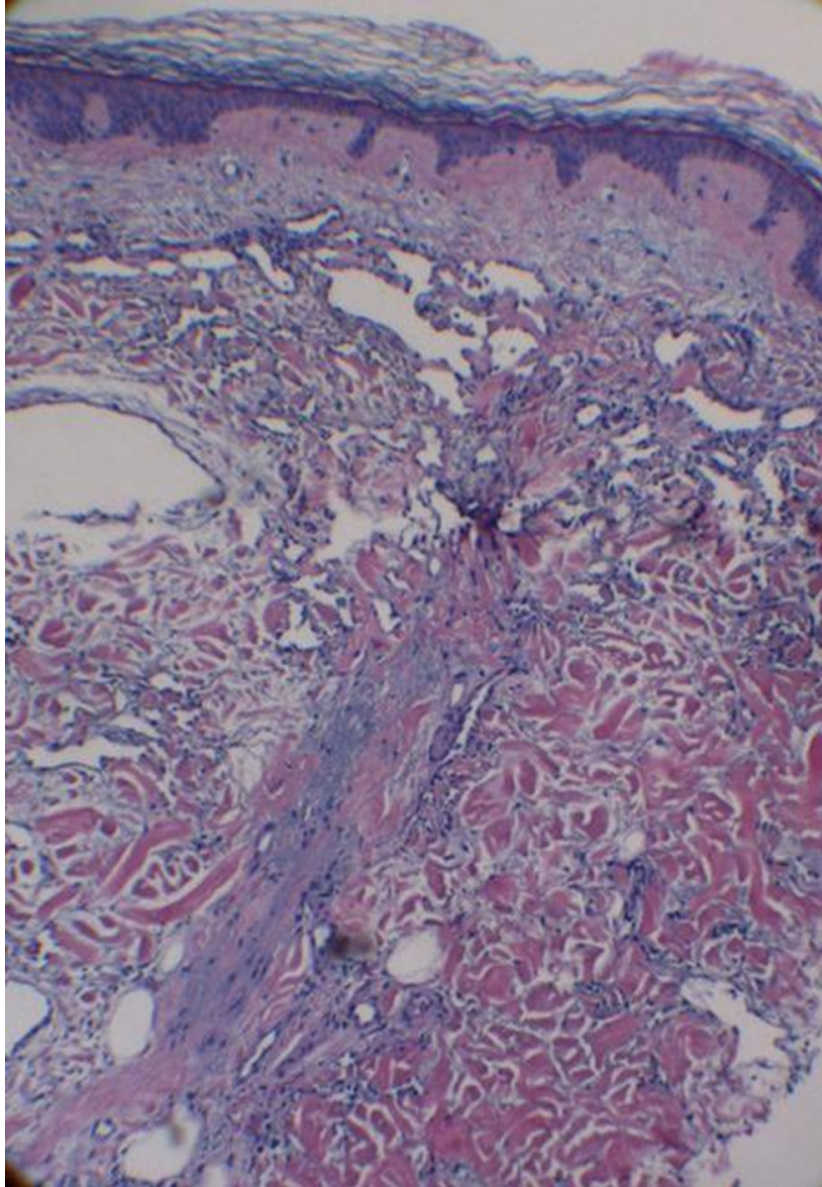

Figure 3. Angiosarcome cutané avec structures vasculaires nombreuses disséquant le derme (hématoxyline et éosine, $\times 4$ ).

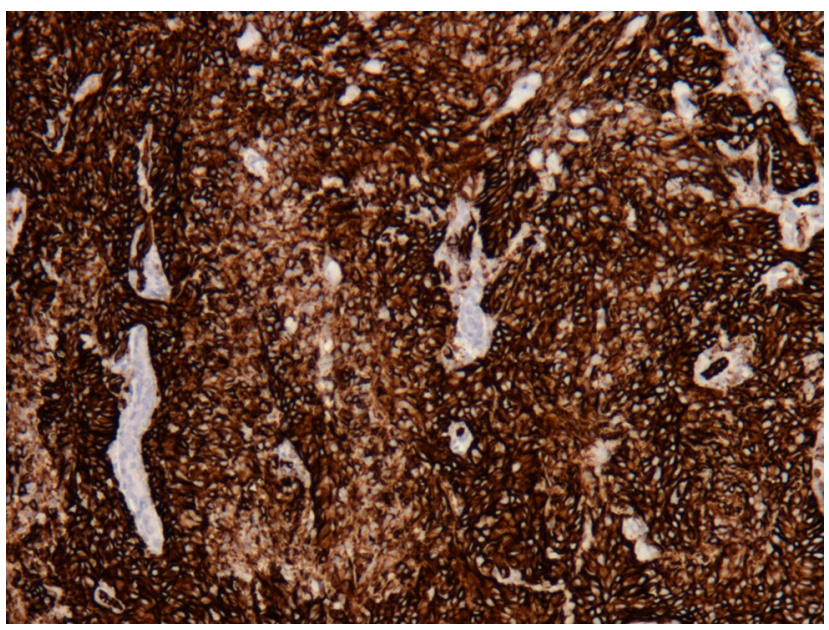

Figure 4. Marquage CD31 fortement positif $(\times 10)$.

Dans le cas précis des AStc, aucun facteur n'a été réellement identifié [4]. Le rôle du soleil est discuté car l'AStc n'est pas l'apanage des patients avec une alopécie [4]. L'AStc se présente sous la forme d'une lésion cutanée ecchymotique, qui peut être prise à tort pour une lésion bénigne ou un banal hématome [1] et qui s'étend de façon centrifuge rapidement jusqu'à affecter une large surface cutanée. Dans les formes les plus avancées, on retrouve des nodules surélevés, une infiltration tissulaire palpable, des ulcérations cutanées, voire des hémorragies $[1,4]$. Des lésions satellites à proximité de la lésion initiale peuvent être observées [4]. L'AStc est souvent multifocal avec une extension plus importante que la clinique ne laisse entrevoir. Notons parfois des formes trompeuses, à l'image d'un patient dont la nappe cutanée angiomateuse était mieux visible tête inclinée [5]. L'histoire de notre patiente illustre bien cette cinétique particulière avec une lésion anodine d'expansion rapidement progressive. Le diagnostic est histologique avec la mise en évidence de vaisseaux capillaires sanguins ou lymphatiques, de taille et de forme variables avec une disposition anarchique. La lésion est souvent mal délimitée et dissèque le derme [2]. Les AS peuvent montrer divers degrés de différenciation et peuvent faire évoquer d'autres diagnostics (mélanome, carcinome, fibrosarcome). Compte tenu des degrés de différenciation variable, il est conseillé au cours des AS de faire un panel assez large de marqueurs vasculaires car certains peuvent être négatifs.

Les immunomarquages CD31 et CD34 doivent être systématiques [2]. D'autres marqueurs (facteur VIII, lectine) peuvent être demandés [4]. Le marquage CD31 est constamment et diffusément positif alors que le CD34 peut être négatif comme dans notre cas [2].

Le pronostic des AS est péjoratif. La survie à 5 ans des AS est de 12 à $35 \%$ [1,4]. Un âge avancé, une tumeur de plus de $5 \mathrm{~cm}$, des métastases au diagnostic ou un état général altéré seraient des facteurs de mauvais pronostic [1]. Les récidives locales (35 à $86 \%$ des cas) ou à distance, habituellement ganglionnaires cervicales et pulmonaires ( $50 \%$ des cas), sont très fréquentes même après un traitement bien conduit du cancer primitif. Le traitement consiste en une chirurgie large associée à une radiothérapie adjuvante (50-60 Gy) [1]. La radiothérapie seule est insuffisante. Cependant, un tiers des patients présentent une maladie métastatique ou inopérable au diagnostic [6]. En cas de forme inopérable, le traitement repose sur une chimiothérapie par anthracyclines ou taxanes [1,6-8], avec une survie sans récidive entre 4 et 9,5 mois. Les chimiothérapies peuvent être limitées en raison de l'âge avancé et de comorbidités associées. Avec le développement des thérapies ciblées en oncologie, l'utilisation du bévacizumab, un anticorps recombinant humanisé anti-VEGF, a été proposée. Le VEGF est en effet un marqueur angiogénique important des AS [1]. Le bévacizumab a été utilisé en monothérapie, en association avec la radiothérapie ou en combinaison avec une chimiothérapie, le paclitaxel notamment [6]. L'association bévacizumab-paclitaxel est parfois associée à des réponses objectives et des survies prolongées en comparaison à la chimiothérapie seule. Cependant, les grandes séries ne confirment pas cette notion [9]. Il faut noter que ces dernières incluent différents types d'AS, et pas seulement les AStc. D'autres anti-angiogéniques ont également été étudiés (sorafenib, sunatinib ou pazopanib) [6].

\section{Conclusion}

L'AStc est une tumeur rare du sujet âgé pour lequel le traitement curatif reste la chirurgie avec de larges marges d'exérèse accompagnée d'une radiothérapie. Il est 
important de rester vigilant devant toute tache à type de macule ecchymotique de l'extrémité céphalique qui ne guérit pas rapidement ou qui s'étend.

\section{Déclaration de liens d'intérêts}

Les auteurs déclarent ne pas avoir de liens d'intérêts.

\section{Références}

[1] Young RJ, Brown NJ, Reed MW, et al. Angiosarcoma. Lancet Oncol 2010;11(10):983-91.

[2] Guinebretière JM. Tumeurs et pseudotumeurs vasculaires. Cas $\mathrm{n}^{\circ}$ 5. Angiosarcome bien différencié. Ann Pathol 2011;31(4):276-80.

[3] Wilson-Jones E. Malignant angioendothelioma of the skin. $\mathrm{Br} \mathrm{J}$ Dermatol 1964;76:21-39.
[4] Requena L, Sangueza OP. Cutaneous vascular proliferations. Part III. Malignant neoplasms, other cutaneous neoplasms with significant vascular component, and disorders erroneously considered as vascular neoplasms. J Am Acad Dermatol 1998;38(2 Pt 1):143-75 [quiz 176-8].

[5] Dagrégorio G, Levillain P, Guillet G. Angiosarcome du cuir chevelu : une présentation clinique inhabituelle. Ann Dermatol Venereol 2005;132(12 Pt 1):1038-9.

[6] Becquart 0 , Girard $C$, Lesage $C$, et al. Angiosarcomes cutanés de la tête et du cou inopérables traités par bévacizumab et paclitaxel. Ann Dermatol Venereol 2018;145(6-7):451-3.

[7] Ockenfels HM, Brockmeyer NH, Hengge U, et al. Cutaneous angiosarcoma: a novel therapy with liposomal doxorubicin? J Eur Acad Dermatol Venereol 1996;6:71-5.

[8] Penel N, Bui BN, Bay JO, et al. Phase II trial of weekly paclitaxel for unresectable angiosarcoma: the ANGIOTAX Study. J Clin Oncol 2008;26(32):5269-74.

[9] Ray-Coquard IL, Domont J, Tresch-Bruneel E, et al. Paclitaxel given once per week with or without bevacizumab in patients with advanced angiosarcoma: a randomized phase II trial. J Clin Oncol 2015;33(25):2797-802. 